\title{
Comparison of Refolding Activities between Nanogel Artificial Chaperone and
}

\section{GroEL Systems}

Wakiko Asayama $^{\text {a, b }}$, Shin-ichi Sawada ${ }^{\text {a }}$, Hideki Taguchi ${ }^{\text {c }}$, and Kazunari Akiyoshi ${ }^{\text {a, b, * }}$

a Institute of Biomaterials \& Bioengineering, Tokyo Medical \& Dental University 2-3-10,

Kanda-surugadai, Chiyoda-ku, Tokyo 101-0062, JAPAN

b Center of Excellence Program for Frontier Research on Molecular Destruction and

Reconstruction of Tooth and Bone, Tokyo Medical and Dental University, Tokyo, Japan.

c Department of Medical Genome Sciences, Graduate School of Frontier Sciences, The

University of Tokyo 5-1-5, Kashiwanoha, Kashiwa-shi, Chiba, 277-8562, Japan

${ }^{*}$ Corresponding author.

Tel.: +81-3-5280-8020; fax: +81-3-5280-8027, E-mail address: akiyoshi.org@ $@$ tmd.ac.jp (Akiyoshi, K.) 


\begin{abstract}
The chaperone-like activity of a nanogel of cholesteryl group-bearing pullulan (CHP) was compared with that of GroEL for refolding acid-denatured green fluorescent protein (GFP). The refolding of denatured GFP was carried out by dilution of acid-denatured GFP in the presence of the nanogel or GroEL. GFP fluorescence was increasingly repressed with increases in the concentration of the CHP nanogel or GroEL added to the dilution buffer. The concentrations of $50 \%$ inhibition of recovery of GFP fluorescence were $0.03 \mu \mathrm{M}$ (GroEL) and $0.08 \mu \mathrm{M}$ (CHP nanogel), respectively. The refolding was resumed by the addition of ATP into the GroEL ( 0.20 $\mu \mathrm{M})$ system or by the addition of methyl- $\beta$-cyclodextrin into the nanogel $(0.20 \mu \mathrm{M})$ system. In the nanogel-GFP system, about $90 \%$ of the intensity was recovered within 10 min. The half time $\left(t_{1 / 2}\right)$ for refolding in the CHP nanogel system $(36 \mathrm{sec})$ is almost equal to that of the natural chaperone GroEL-GroES system.
\end{abstract}

Keywords:

Molecular chaperone; Cholesteryl group-bearing pullulan; Protein refolding 


\section{Introduction}

There is an obvious need for and much interest in producing large amounts of biologically active recombinant proteins, both for medical applications as well as basic protein science. In many cases, however, there are problems with the aggregation and misfolding of the proteins synthesized [1-3].

In living system, molecular chaperones selectively trap heat-denatured proteins or their intermediates in refolding to prevent their irreversible aggregation. Then, with the aid of ATP and another co-chaperone, the host chaperone releases the protein in its refolded form. The chaperonin GroEL-GroES is a well-known molecular chaperone in Escherichia coli [4-6]. Functional GroEL is composed of 14 identical $60-\mathrm{kDa}$ subunits arranged in a bilayer of sevenmembered rings and acts as a host which can selectively interact with nascent peptide chains (guest) mainly by hydrophobic interaction to minimize off-pathway interactions. The host-guest interaction is controlled by a cyclic change in the conformation of GroEL upon the binding of ATP and GroES. This is an excellent example of a molecular nano-machine [7-9].

Molecular chaperone systems have inspired to explore new concepts for protein refolding. [10-15]. We designed an amphiphilic nanogel that behaves as a host for a guest protein to simulate the function of molecular chaperones. In particular, we developed a novel artificial chaperone system using nanogels of cholesteryl group-bearing pullulan (CHP) [16-19]. We 
reported chaperone-like activity of CHP nanogels in the refolding of heat denatured proteins and chemically denatured proteins, and in the inhibition of protein aggregation (i.e., carbonic anhydrase, citrate synthease, and amyloid $\beta$-protein) [20-23]. Denatured proteins trapped in the nanogels were refolded in high yields upon the dissociation of gel structure by the addition of cyclodextrins (CDs). We report here direct comparison of the refolding activity of aciddenatured green fluorescent protein (GFP) in the same conditions for the artificial chaperone, CHP nanogel system and a natural chaperone, GroEL system. GFP is widely used as a very convenient tool for studying gene expression and protein localization. GFP is also a convenient protein for the study of in vitro protein folding. Folding of GFP can be easily and directly monitored in real time by measuring fluorescence [24-30]. The behavior of refolding in the CHP nanogel was very similar to that of the GroEL system.

\section{Material and methods}

\subsection{Materials}

Cholesteryl group-bearing pullulan (CHP) was synthesized as reported previously from a pullulan of molecular weight 100,000. It contains 1.2 cholesteryl groups per 100 glucose units [16-19]. Green fluorescent protein (GFP) from the jellyfish Aequorea victoria, and GroEL and 
GroES from Escherichia coli, were purified as described [27,31]. M- $\beta$-CD was purchased from Tokyo Chemical Industry Co. Ltd. Trizma base and ATP were purchased from Sigma-Aldrich Japan, Tokyo, Japan. Other chemicals were of analytical grade and used without further purification. The concentrations of proteins were determined spectrophotometrically at $280 \mathrm{~nm}$ using the following extinction coefficients: $20,600 \mathrm{M}^{-1} \mathrm{~cm}^{-1}$ for GFP, $0.163\left(\mathrm{mg} \mathrm{mL}^{-1} \text { protein }\right)^{-1}$ $\mathrm{cm}^{-1}$ for GroEL, and 9,000 $\mathrm{M}^{-1} \mathrm{~cm}^{-1}$ for GroES.

\subsection{Refolding analysis of acid-denatured GFP}

Refolding analysis of acid-denatured GFP was performed as in a previous study [27]. 10 $\mu \mathrm{M}$ GFP solution ( $8 \mathrm{mM}$ Tris-HCl, $0.3 \mathrm{mM}$ EDTA, $1 \mathrm{mM}$ DTT, $\mathrm{pH}$ 7.5) was mixed with the same volume of $66 \mathrm{mM} \mathrm{HCl}$ and incubated for $1 \mathrm{~min}$ to give denatured GFP solution (final 5 $\mu \mathrm{M}, \mathrm{pH} 1.5)$. Denatured GFP solution $(5 \mu \mathrm{M})$ was diluted into a dilution buffer $(50 \mathrm{mM}$ MOPS-NaOH, $100 \mathrm{mM} \mathrm{KCl}, 5 \mathrm{mM} \mathrm{Mg}\left(\mathrm{CH}_{3} \mathrm{COO}\right)_{2}, 5 \mathrm{mM}$ DTT, pH 7.0) containing CHP nanogel, GroEL, or GroEL plus GroES (final $0.05 \mu \mathrm{M}$ GFP), and the mixture was incubated for $380 \mathrm{sec}$. After $380 \mathrm{sec}$ from the time of dilution, ATP solution was added into the mixture with GroEL or GroEL/GroES (final $1 \mathrm{mM}$ ATP). Similarly, $\mathrm{m}-\beta-\mathrm{CD}$ solution was added into the mixture with CHP nanogels (final $3.7 \mathrm{mM} \mathrm{m- \beta -CD).} \mathrm{The} \mathrm{recovery} \mathrm{of} \mathrm{GFP} \mathrm{fluorescence} \mathrm{was}$ monitored continuously with a fluorometer FP-750 (JASCO) (excitation at $398 \mathrm{~nm}$ and 
emission at $508 \mathrm{~nm}$ ). All experiments were performed at $25^{\circ} \mathrm{C}$.

2.3 Size exclusion chromatography (SEC)-analysis of nanogels and nanogel-GFP complexes

SEC sample solutions, native GFP, CHP nanogel, GFP-CHP nanogel complex, and GFP-

CHP nanogel complex plus m- $\beta-\mathrm{CD}$, were prepared. Acid-denatured GFP solution (denatured at $\mathrm{pH} 1.5$, for $1 \mathrm{~min}$ ) was diluted 100-fold in CHP nanogel solution, and incubated for $20 \mathrm{~min}$ at $25^{\circ} \mathrm{C}$ (GFP-CHP nanogel complex). Furthermore, $\mathrm{m}-\beta$-CD was added to the GFP-CHP nanogel complex solution after $380 \mathrm{sec}$ from the time of dilution, and incubated for $20 \mathrm{~min}$ (GFP-CHP nanogel complex plus $\mathrm{m}-\beta$-CD). Each of the final concentrations were $0.05 \mu \mathrm{M}$ GFP, $0.80 \mu \mathrm{M}$ CHP nanogel, and $3.73 \mathrm{mM} \mathrm{m}-\beta-\mathrm{CD}$. These sample solutions $(20 \mu \mathrm{L})$ were applied to the SEC column (TSK gel Super SW 3000 column, Tosoh). GFP was detected by a FP-2025 plus FP detector $(\mathrm{JASCO})$ at $35{ }^{\circ} \mathrm{C}, \lambda \mathrm{ex}=398 \mathrm{~nm}, \lambda \mathrm{em}=508 \mathrm{~nm}$. The elution buffer for all examinations was $50 \mathrm{mM}$ MOPS- $\mathrm{NaOH}(\mathrm{pH} 7.0), 100 \mathrm{mM} \mathrm{KCl}, 5.0 \mathrm{mM} \mathrm{Mg}\left(\mathrm{CH}_{3} \mathrm{COO}\right)_{2}$ • $4 \mathrm{H}_{2} \mathrm{O}, 5.0 \mathrm{mM}$ DTT. Elution rate was $0.5 \mathrm{~mL} \mathrm{~min}^{-1}$.

\section{Results}

3.1. Interaction between acid-denatured GFP and GroEL or the CHP nanogel 
The interactions of GroEL and CHP nanogels with acid-denatured GFP were investigated.

GFP $(5 \mu \mathrm{M})$ was denatured at $\mathrm{pH} 1.5$ for $1 \mathrm{~min}$ at $25^{\circ} \mathrm{C}$. The GFP fluorescence disappeared due to the denaturation. Refolding of denatured GFP was carried out by dilution of denatured GFP with dilution buffer $\left(\mathrm{pH} 7.0,25{ }^{\circ} \mathrm{C}\right)(100$ times dilution, final concentration, $0.05 \mu \mathrm{M})$. The refolding of GFP was monitored by the recovery of GFP fluorescent intensity. The recovery of GFP fluorescence was $~ 70 \%$ (Fig. 1, "spont.") in the absence of chaperones.

In the presence of GroEL in dilution buffer, spontaneous refolding was inhibited depending on the concentration of GroEL (Fig. 1a). From the results of Fig. 1a, the repression rates of GFP fluorescence were plotted as a function of various concentrations of GroEL in dilution buffer (Fig. 1c). When the concentration of GroEL was more than $0.1 \mu \mathrm{M}$, the GFP fluorescence was suppressed to less than $10 \%$. These results indicate that GroEL trapped acid-denatured GFP as reported previously [27].

The same experiment was carried out using CHP nanogels instead of GroEL (Fig. 1b and 1c). Similar to the GroEL system, GFP fluorescence was gradually repressed with increases in the concentration of the CHP nanogel added to the dilution buffer. When the concentration of the CHP nanogel reached more than $0.20 \mu \mathrm{M}$, the fluorescence was suppressed to less than $10 \%$. The concentrations of $50 \%$ inhibition of recovery of GFP fluorescence were $0.03 \mu \mathrm{M}$ (GroEL) and $0.08 \mu \mathrm{M}$ (CHP nanogel), respectively. 
The intensity of native GFP $(0.05 \mu \mathrm{M})$ fluorescence did not change over the 1 h period after the addition of GroEL $(0.80 \mu \mathrm{M})$ or CHP nanogel $(0.80 \mu \mathrm{M})$. It is known that GroEL does not bind native GFP [27]. The data suggest that CHP nanogel did not bind native GFP, similar to the result with GroEL.

\subsection{Refolding of Acid-denatured GFP assisted by chaperones}

The refolding of GFP was estimated by real time monitoring under the same conditions of the recovery of fluorescence in the GroEL and nanogel systems (Fig. 2). After acid-denatured GFP was diluted 100-fold into the dilution buffer with $0.20 \mu \mathrm{M}$ GroEL or $0.20 \mu \mathrm{M}$ CHP nanogel, the intensity of GFP fluorescence was suppressed to less than $10 \%$ in both systems, as described above. Under this condition, most of the denatured-GFPs were trapped by GroEL or the CHP nanogel. Refolding was resumed by the addition of ATP into the GroEL system. After the addition of ATP (final $1.0 \mathrm{mM}$ ) into this diluted solution, GFP refolding started. After 600 sec, the intensity reached $86 \%$, which was above the percentage of spontaneous refolding $(\sim 70 \%)$. GroEL can assist in overall refolding by preventing the aggregation of denatured GFP by trapping it. GroES plays an important role in the refolding of proteins in the GroEL system. In the presence of a GroEL $(0.20 \mu \mathrm{M})-\operatorname{GroES}(0.20 \mu \mathrm{M})$ system in dilute buffer, the intensity of GFP fluorescence was suppressed to less than $10 \%$. In the trapping process of GroEL with acid-denatured GFP, GroES did not exhibit any affect at all. However, when ATP was added to the mixture of GroEL-GroES and denatured GFP, the rate of refolding was drastically accelerated in comparison with that of the GroEL system alone.

In the CHP nanogels-GFP system, approximately $90 \%$ of the intensity was recovered within 10 min after the addition of $\mathrm{m}-\beta-\mathrm{CD}$ (final $3.73 \mathrm{mM}$ ). The refolding half time $\left(\mathrm{t}_{1 / 2}\right)$ in the 
CHP nanogel system is $36 \mathrm{sec}$. This value is almost equal to that of the natural chaperone GroEL-GroES system. Fig. 3 shows the result of acid-denatured GFP refolding with various concentrations of GroEL or the CHP nanogel after the same dilution experiments as shown in Fig. 2. The behavior of refolding in the CHP nanogel was very similar to that of the GroEL system. For example, in the presence of $0.01 \mu \mathrm{M}$ GroEL or $0.04 \mu \mathrm{M}$ nanogel, the recovery of fluorescent intensity was almost the same as that of spontaneous refolding ( 70\%). However, after the addition of ATP or $\mathrm{m}-\beta-\mathrm{CD}$, the intensity recovered to more than $90 \%$. This indicates that both chaperones selectively trapped more than $20 \%$ of the GFP that tends to be easily aggregated, and assisted refolding by a chaperoning effect.

Fig. 4 shows the size exclusion chromatograms that were detected by GFP fluorescence in the various conditions. After dilution of acid denatured GFP with the CHP nanogel, GFP was trapped to the nanogel in a nonfluorescent form (Fig. 4c). The CHP nanogel dissociated in the presence of $\mathrm{m}-\beta-\mathrm{CD}$ and GFP was released (Fig. $4 \mathrm{~d}$ ).

\section{Discussion}

There are many reports on the mechanism of GroEL-assisted refolding [27,29,32,33]. Various sized non-native proteins interact with hydrophobic apical domains of GroEL, mainly by hydrophobic interaction [34]. In the presence of GroES and ATP, GroEL undergoes conformational change. Subsequently, a non-native protein is encapsulated into the GroEL cavity for proteins smaller than $60 \mathrm{kDa}$, such as GFP. In this cavity, which is isolated in the hydrophilic environment, the folding of non-native protein can occur without aggregation. It is 
important that only one acid-denatured GFP $(27 \mathrm{kDa})$ can be trapped and encapsulated into the GroEL cavity. This property is indispensable to protect against the aggregation of proteins and refolding in the isolated microenvironment [6,35]. Release of the encapsulated protein results in ATP hydrolysis and the binding of GroES to the trans-ring of GroEL (Fig. 5a). Active GroEL that can bind denatured protein is regenerated by the hydrolysis of ATP and rebinding of ATP. In the presence of excess GroEL, the apparent rate of refolding tends to decrease (Fig. 3b). The reason of this inhibition is not known well. The released protein that is not yet refolded is probably caught again by GroEL and leads to following cyclic process (Fig.5 a) [35,36]. The refolding of the protein delays due to the recycling process.

CHP nanogel is a monodispersible hydrogel nanoparticle $(-20 \mathrm{~nm})$ which is formed in water by the self-assembly of cholesteryl group-bearing pullulans (approximately 4 CHP polymers per nanogel). Amphiphilic CHP nanogels have 4-5 non-covalently cross-linking points that consist of cholesteryl groups. CHP polymers occupy $10 \%$ of the volume of the nanogel, and water interspaces the rest. These structural characteristics of CHP nanogels make it possible for them to interact with proteins. The CHP nanogels effectively complexed with the denatured forms of carbonic anhydrase $\mathrm{B}(\mathrm{CAB})$ such as a heat-denatured form above its denaturation temperature $\left(55^{\circ} \mathrm{C}\right)$ or in a chemically denatured form by guanidine hydrochloride [20,21]. We report here that acid denatured GFP also effectively interacted with the CHP nanogel. The CHP 
nanogel bound strongly with denatured proteins due to the hydrophobic interaction between hydrophobic-associated domains of the choresteryl groups and the hydrophobic surface of the denatured protein. In living systems, molecular chaperones are known to trap denatured proteins selectively. Simple amphiphilic nanogels can simulate this function.

The dynamics of molecular chaperones is controlled by the binding of suitable effectors such as ATP and co-chaperones (Fig. 5a). In our artificial system, CDs were used as modulators of the structure of nanogels. The cross-linking domains, which consisted of hydrophobic interaction between cholesteryl groups in the nanogels, become disrupted by the encapsulation of a cholesteryl moiety into a CD cavity [22]. The dissociation of the nanogel by the addition of $m-\beta-C D$ caused an attenuation of the interaction between the nanogel and the denatured protein (Fig. 5b). Thus, protein folding is predicted to start under the more hydrophilic environment. This is similar to the two-step mechanism of a molecular chaperone; i.e., capture of a denatured protein and release of the refolded protein (Fig. 5).

In conclusion, the CHP nanogel assisted the refolding of acid-denatured GFP by a mechanism similar to that of GroEL-ATP system. Well-defined physically cross-linked amphiphilic nanosized gel (nanogel) with a size comparable to that of proteins is useful for effective trapping of proteins inside hydrogel nano-matrix so as to prevent protein aggregation. $\mathrm{CD}$ is an effecter molecule that controls the binding ability of these host nanogels to proteins 
like ATP in the GroEL system. The chaperon-like activity of the CHP nanogel, an artificial chaperone, for acid denatured GFP, is comparable to that of the native chaperone GroEL. Aggregation was inhibited by the trapping of denatured proteins into the isolated nanospace and the releasing/refolding of the denatured protein was controlled by the changing amphiphilicity of the chaperones.

\section{Acknowledgements}

This work was supported by Grants-in-Aid for Scientific Research from the Japanese Government (No. 17300147, 18011002) and CREST, JST. Pacific Edit reviewed the manuscript prior to submission.

\section{References}

[1] D. D. B. Clark, Current Opinion in Biotechnology 9 (1998) 157-163.

[2] F. Baneyx, Current Opinion in Biotechnology 10 (1999) 411-421.

[3] A. Horwich, J. Clin. Invest. 110 (2002) 1221-1232.

[4] B. Bukau, A. L. Horwich, Cell 92 (1998) 351-366.

[5] F. U. Hartl, M. Hayer-Hartl, Science 295 (2002) 1852-1858.

[6] Z. Xu, A. L. Horwich, P. B. Sigler, Nature 388 (1997) 741-750.

[7] W. A. Fenton, A. L. Horwich, Protein Science 6 (1997) 743-760.

[8] P. B. Sigler, X. Zhaohui, H. S. Rye, S. G. Burston, W. A. Fenton, A. L. Horwich, Annu. Rev. 
Biochem. 67 (1998) 581-608.

[9] R. J. Ellis, Curr. Biol. 11 (2001) R1038-1040.

[10] D. Rozema, S. H. Gellman, J. Am. Chem. Soc., 117 (1995), 2373-2374.

[11] S. Machida, S. Ogawa, S. Xiaohua, T. Takaha, K. Fujii, K. Hayashi, FEBS Letters 486 (2000) 131-135.

[12] N. Yoshimoto, T. Hashimoto, M. M. Felix, H. Umakoshi, R. Kuboi, Biomacromolecules 4 (2003) 1530-1538.

[13] H. Lanckriet, A. P. J. Middelberg, Biotechnol. Prog. 20 (2004) 1861-1867.

[14] D. Lu, K. Zhang, Z. Liu, Biochem. Eng. J. 25 (2005) 141-149.

[15] R. Yazdanparast, R. Khodarahmi, Int. J. Biol. Macromol. 40 (2007) 319-326.

[16] K. Akiyoshi, S. Deguchi, N. Moriguchi, S. Yamaguchi, J. Sunamoto, Macromolecules 26 (1993) 3062-3068.

[17] K. Akiyoshi, J. Sunamoto, Supramol. Sci. 3(1996) 157-163.

[18] K. Akiyoshi, S. Deguchi, H. Tajima, T. Nishikawa, J. Sunamoto, Macromolecules 30 (1997) 857-861.

[19] K. Kuroda, K. Fujimoto, J. Sunamoto, K. Akiyoshi, Langmuir 18 (2002) 3780-3786.

[20] K. Akiyoshi, Y. Sasaki, J. Sunamoto, Bioconjugate Chem. 10 (1999) 321-324.

[21] Y. Nomura, M. Ikeda, Y. Yamaguchi, Y. Aoyama, K. Akiyoshi, FEBS Letters 553 (2003) 
271-276.

[22] Y. Nomura, Y. Sasaki, M. Takagi, T. Narita, Y. Aoyama, K. Akiyoshi, Biomacromolecules 6 (2005) 447-452.

[23] K. Ikeda, T. Okada, S. Sawada, K. Akiyoshi, K. Matsuzaki, FEBS Letters 580 (2006) 65876595.

[24] S. H. Bokman, W. W. Ward, Biochem. Biophys. Res. Commun. 101 (1981) 1372-1380.

[25] W. W. Ward, S. H. Bokman, Biochemistry 21 (1982) 4535-4540.

[26] F. Yang, L. G. Moss, G. N. Jr. Phillips, Nat. Biotechnol. 14 (1996) 1246-1251.

[27] Y. Makino, K. Amada, H. Taguchi, M. Yoshida, J. Biol. Chem. 272 (1997) 12468-12474.

[28] R. Y. Tsien, Annu. Rev. Biochem. 67 (1998) 509-544.

[29] T. Makio, M. Arai, K. Kuwajima, J.Mol. Biol. 293 (1999) 125-137.

[30] T. Ueno, H. Taguchi, H. Tadakuma, M. Yoshida, T. Funatsu, Molecular Cell 14 (2004) 423434.

[31] F. Motojima, T. Makio, K. Aoki, Y. Makino, K. Kuwajima, M. Yoshida, Biochem. Biophys. Res. Commun. 267 (2000) 842-849.

[32] J. Buchner, M. Schmidt, M. Fuchs, R. Jaenicke, R. Rudolph, F. X. Schmid, T. Kiefhaber, Biochemistry 30 (1991) 1586-1591.

[33] A. Brinker, G. Pfeifer, M. J. Kerner, D. J. Naylor, F. U. Hartl, M. Hayer-Hartl, Cell 107 
(2001) 223-233.

[34] K. Aoki, H. Taguchi, Y. Shindo, M. Yoshida, K. Ogasahara, K. Yutani, N. Tanaka, J. Biol. Chem. 272 (1997) 32158-32162.

[35] C. Sakikawa, H. Taguchi, Y. Makino, M. Yoshida, J. Biol. Chem. 274 (1999) 21251-21256.

[36] H. Taguchi, J. Konishi, N. Ishii, M. Yoshida, J. Biol. Chem. 266 (1991) 22411-22418.

\section{Captions to Figures}


Fig. 1. Refolding of acid-denatured GFP $(0.05 \mu \mathrm{M})$ in the presence of GroEL (a) and CHP nanogel (b). Recovery of GFP fluorescence intensity as a function of the concentration of GroEL and CHP nanogel (c).

Fig. 2. Refolding of acid-denatured GFP $(0.05 \mu \mathrm{M})$ mediated by CHP nanogel $(0.20 \mu \mathrm{M})$, GroEL $(0.20 \mu \mathrm{M})$ and GroEL $(0.20 \mu \mathrm{M})$-GroES $(0.20 \mu \mathrm{M})$ system.

Fig. 3. Refolding of acid-denatured GFP $(0.05 \mu \mathrm{M})$ mediated by various concentrations of GroEL (a) or CHP nanogel (b) in the same diluted samples as shown in Fig 2.

Fig. 4. SEC analysis of (a) native GFP ( $0.05 \mu \mathrm{M})$, (b) CHP nanogel $(0.80 \mu \mathrm{M})$, (c) GFP-CHP nanogel complex $(0.05 \mu \mathrm{M} / 0.80 \mu \mathrm{M})$, Acid-denatured GFP solution (denatured at $\mathrm{pH} 1.5$, for 1 min) was diluted 100-fold in CHP nanogel solution, and incubated for $20 \mathrm{~min}$ at $25^{\circ} \mathrm{C}$. (d) GFPCHP nanogel complex plus m- $\beta$-CD $(0.05 \mu \mathrm{M}, 0.80 \mu \mathrm{M}$, and $3.7 \mathrm{mM}) . \mathrm{M}-\beta-\mathrm{CD}$ was added in GFP-CHP nanogel complex solution after $380 \mathrm{sec}$ from the time of dilution and incubated for 20 min. Elutions were monitored by an FP detector $\left(\lambda \mathrm{ex}=398 \mathrm{~nm}, \lambda \mathrm{em}=508 \mathrm{~nm}\right.$, at $\left.35^{\circ} \mathrm{C}\right)$.

Fig. 5. Schematic representation of chaperones-assisted refolding

Comparison of Refolding Activities between Nanogel Artificial Chaperone and GroEL Systems Wakiko Asayama, Shin-ichi Sawada, Hideki Taguchi, and Kazunari Akiyoshi 
Fig. 1

a. GroEL

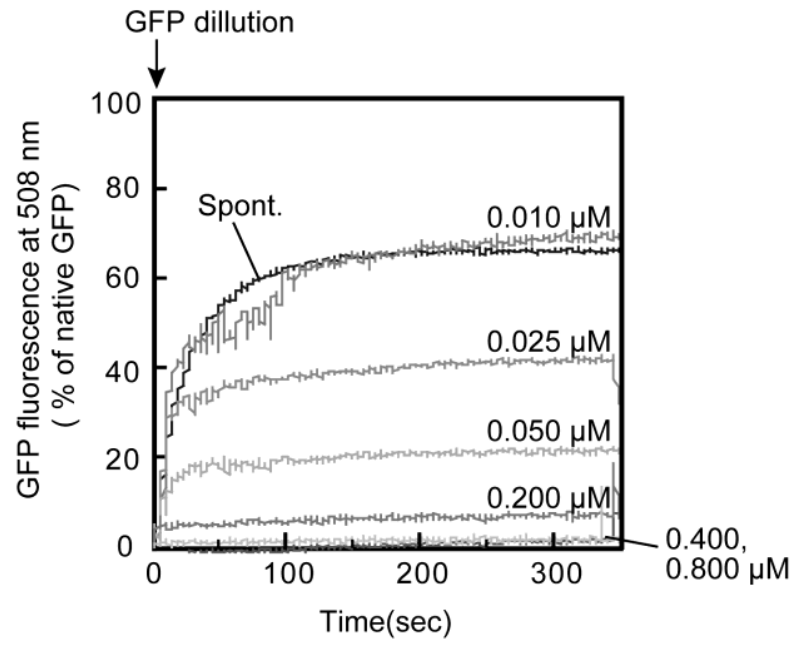

b. CHP nanogel

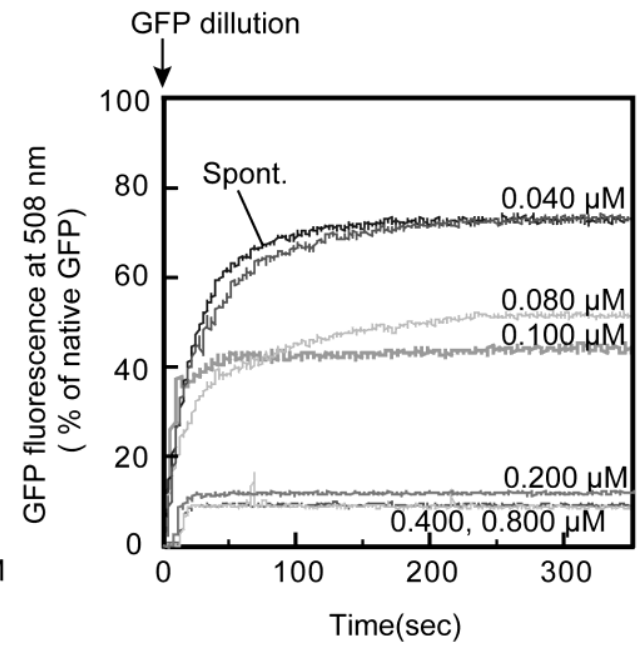

c.

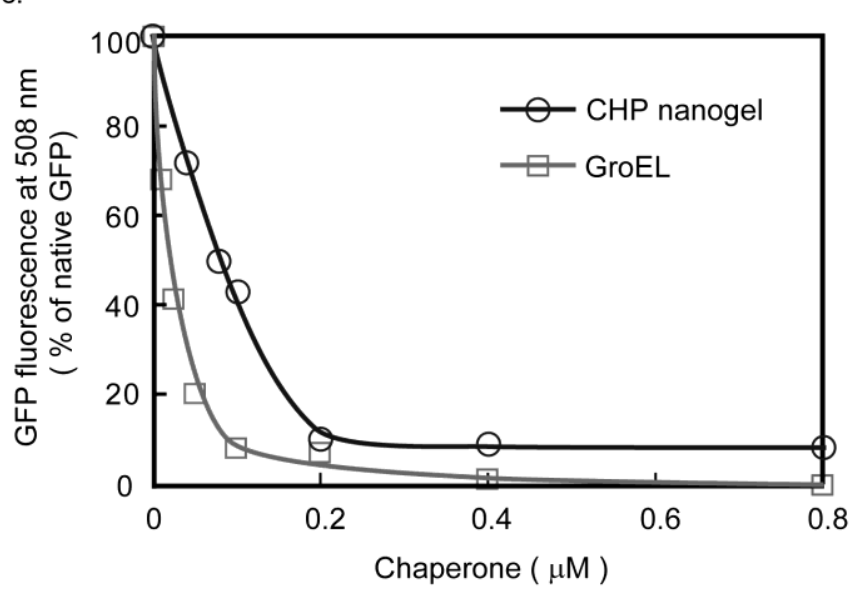

Comparison of Refolding Activities between Nanogel Artificial Chaperone and GroEL Systems Wakiko Asayama, Shin-ichi Sawada, Hideki Taguchi, and Kazunari Akiyoshi 
Fig. 2

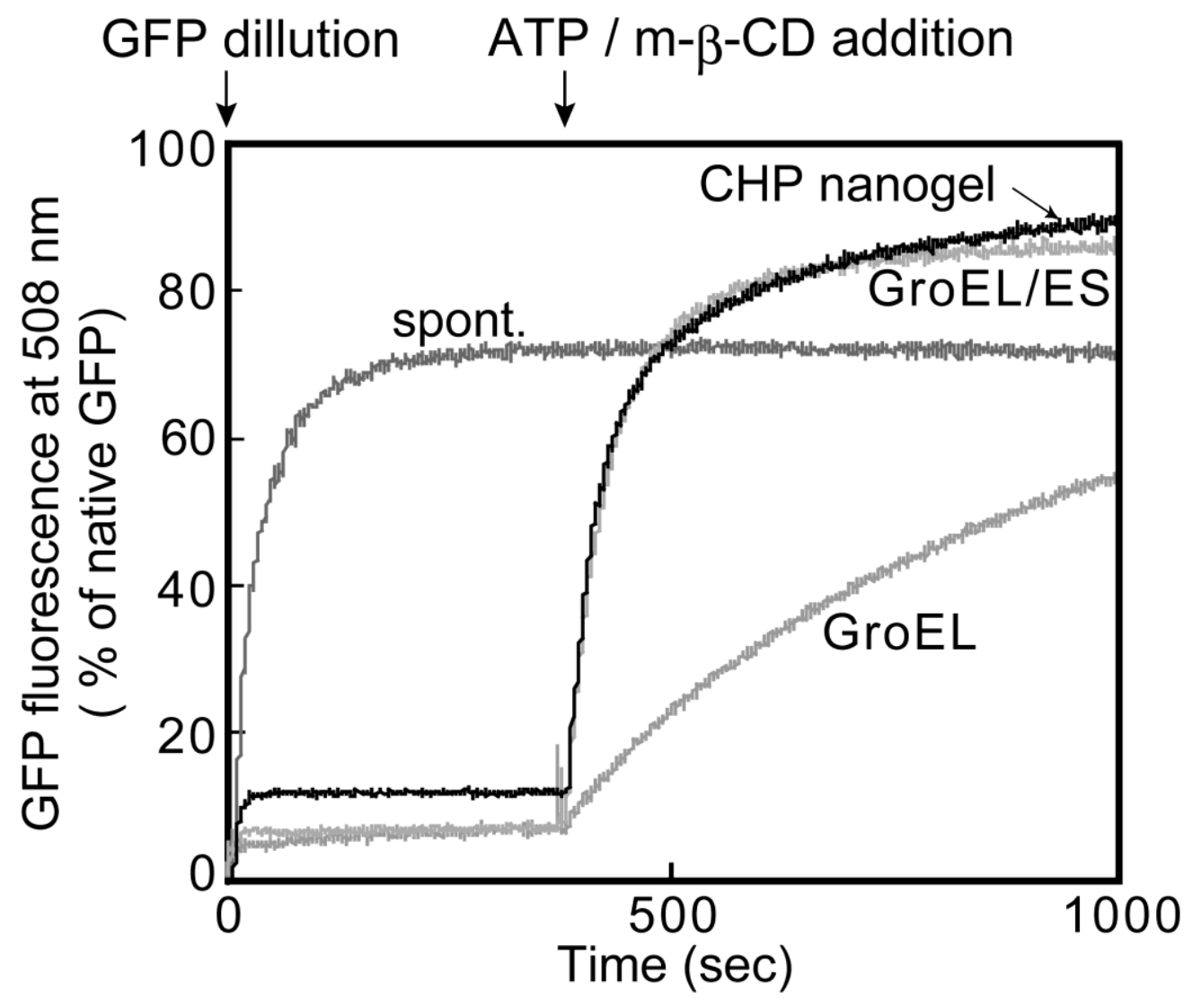

Comparison of Refolding Activities between Nanogel Artificial Chaperone and GroEL Systems Wakiko Asayama, Shin-ichi Sawada, Hideki Taguchi, and Kazunari Akiyoshi 
Fig. 3

a. GroEL

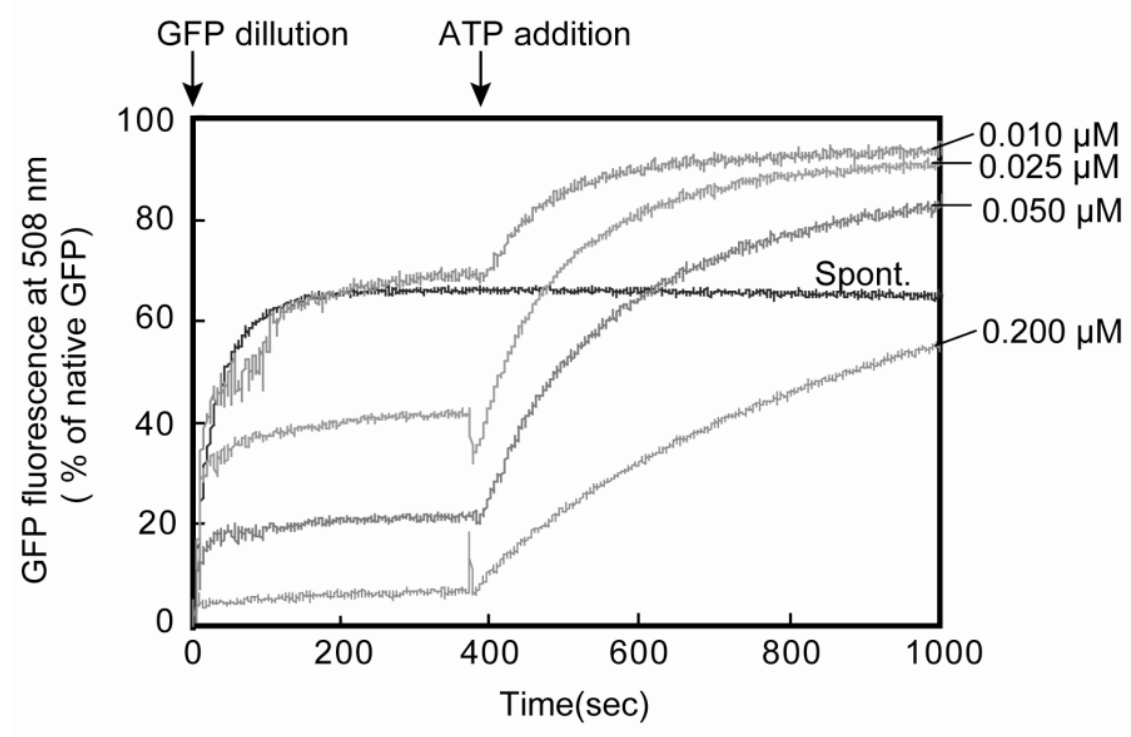

b. CHP nanogel

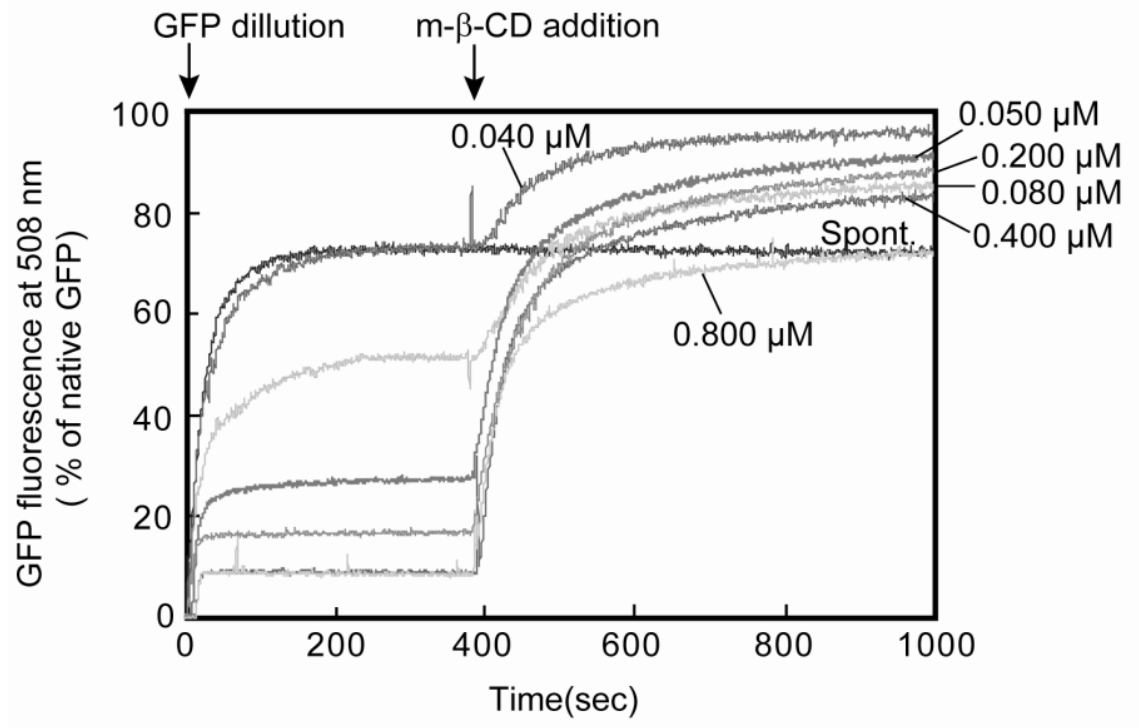

Comparison of Refolding Activities between Nanogel Artificial Chaperone and GroEL Systems 
Wakiko Asayama, Shin-ichi Sawada, Hideki Taguchi, and Kazunari Akiyoshi

Fig. 4

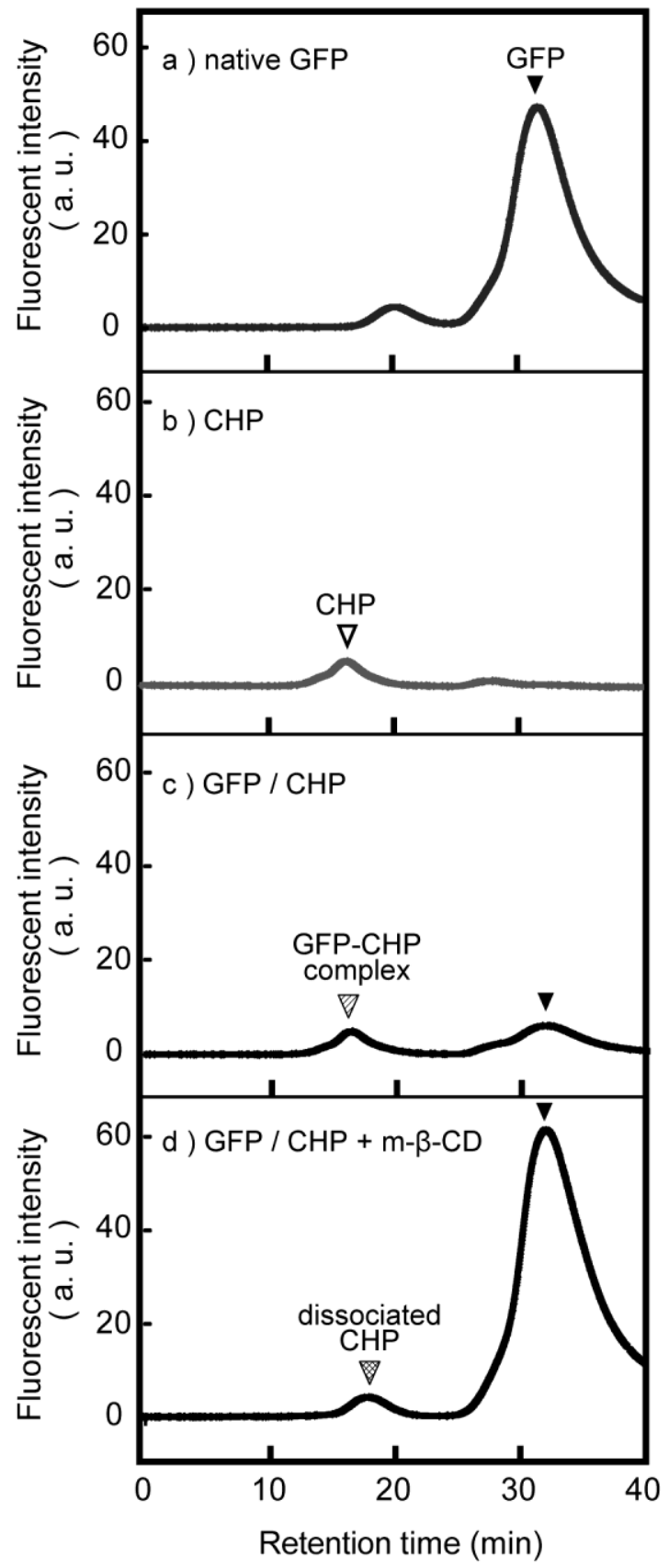

Comparison of Refolding Activities between Nanogel Artificial Chaperone and GroEL Systems 
Wakiko Asayama, Shin-ichi Sawada, Hideki Taguchi, and Kazunari Akiyoshi

Fig. 5

a. GroEL/ES

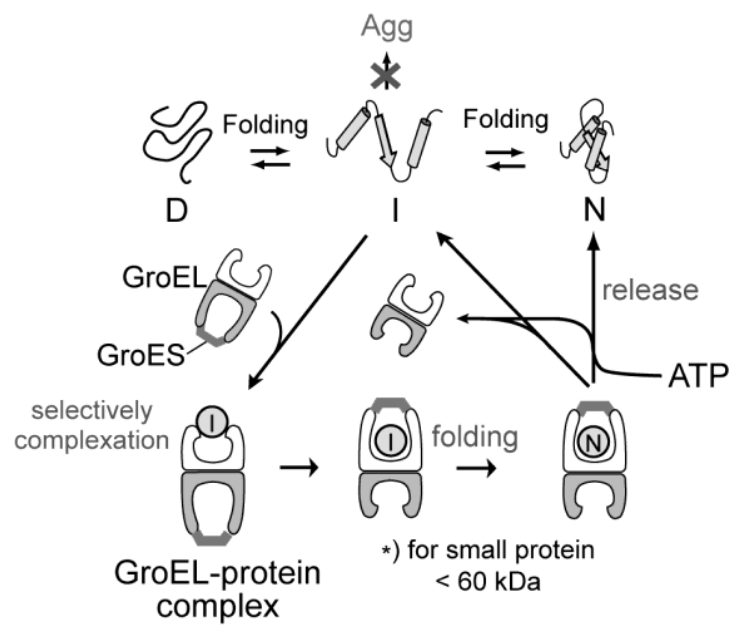

b. CHP-nanogel

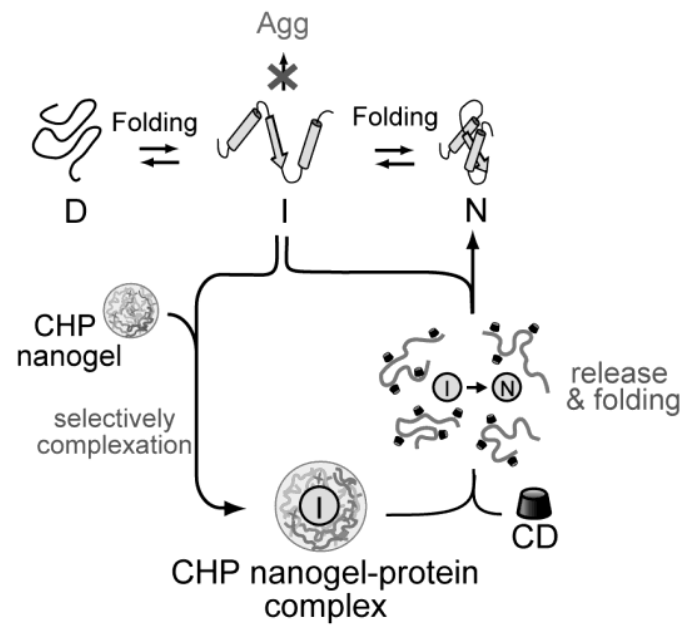

Agg : aggregation, $\mathrm{D}$ : denatured protein, $\mathrm{I}:$ intermediate protein, $\mathrm{N}$ : nature protein 